\title{
BLOW-UP, EXPONENTIAL GROUTH OF SOLUTION FOR A NONLINEAR PARABOLIC EQUATION WITH $p(x)$ - LAPLACIAN
}

\section{AMAR OUAOUA* AND MESSAOUD MAOUNI}

Laboratory of Applied Mathematics and History and Didactics of Mathematics (LAMAHIS) University of 20 August 1955, Skikda, Algeria

${ }^{*}$ Corresponding author: a.ouaoua@univ-skikda.dz

ABSTRACT. In this paper, we consider the following equation

$$
u_{t}-\operatorname{div}\left(|\nabla u|^{p(x)-2} \nabla u\right)+\omega|u|^{m(x)-2} u_{t}=b|u|^{r(x)-2} u .
$$

We prove a finite time blowup result for the solutions in the case $\omega=0$ and exponential growth in the case $\omega>0$, with the negative initial energy in the both case.

\section{Introduction}

We consider the following boundary problem:

$$
\left\{\begin{array}{l}
u_{t}-\operatorname{div}\left(|\nabla u|^{p(x)-2} \nabla u\right)+\omega|u|^{m(x)-2} u_{t}=b|u|^{r(x)-2} u \quad \text { in } \Omega \times(0, T) \\
u(x, t)=0, \quad x \in \partial \Omega, \quad t \geq 0 \\
u(x, 0)=u_{0}(x) \text { in } \Omega
\end{array}\right.
$$

where $\Omega$ is a bounded domain in $\mathbb{R}^{n}, n \geq 1$ with smooth boundary $\partial \Omega$ and $b>0, \omega \geq 0$ are constants, $p($.$) ,$ $m(x)$ and $r($.$) are given measurable functions on \Omega$ satisfying

$$
2 \leq m_{1} \leq m(x) \leq m_{2}<p_{1} \leq p(x) \leq p_{2}<r_{1} \leq r(x) \leq r_{2} \leq p_{*}(x)
$$

Received 2019-04-06; accepted 2019-05-07; published 2019-07-01.

2010 Mathematics Subject Classification. 35K55; 35K61; 35K60 .

Key words and phrases. nonlinear parabolic equation; $p(x)$ - Laplacian; blow-up, exponential grouth.

(C) 2019 Authors retain the copyrights of their papers, and all open access articles are distributed under the terms of the Creative Commons Attribution License. 


$$
\begin{array}{cl}
p_{1}:=e s s_{x \in \Omega} \inf (p(x)), \quad p_{2}:=e s s_{x \in \Omega} \sup (p(x)), \\
r_{1}:=e s s_{x \in \Omega} \inf (r(x)), \quad r_{2}:=e s s_{x \in \Omega} \sup (r(x)), \\
m_{1}:=e s s_{x \in \Omega} \inf (m(x)), \quad m_{2}:=e s s_{x \in \Omega} \sup (m(x)),
\end{array}
$$

and

$$
p_{*}(x)=\left\{\begin{array}{c}
\frac{n p(x)}{e s s \sup _{x \in \Omega}(n-p(x))} \text { if } p_{2}<n \\
+\infty \text { if } p_{2} \geq n
\end{array} .\right.
$$

We also assume that $p(),. m($.$) and r($.$) satisfy the log-Hölder continuity condition:$

$$
|q(x)-q(y)| \leq-\frac{A}{\log |x-y|}, \text { for a.e. } x, y \in \Omega, \text { with }|x-y|<\delta
$$

$A>0,0<\delta<1$

Equation (1.1) can be viewed as a generalization of the evolutional $p$-Laplacian equation

$$
u_{t}-\operatorname{div}\left(|\nabla u|^{p-2} \nabla u\right)+\omega|u|^{m-2} u_{t}=b|u|^{r-2} u
$$

with the constant exponent of nonlinearity $p, m, r \in(2, \infty)$, which appears in various physical contexts. In particular, this equation arises from the mathematical description of the reaction-diffusion/ diffusion, heat transfer, population dynamics processus, and so on (see [11]) and references therein). Recently in [1], in the case $\omega=0$, Agaki proved an existence and blow up result for the initial datum $u_{0} \in L^{r}()$. Otani [17] studied the existence and the asymptotic behavior of solutions of (1.1) and overcome the difficulties caused by the use of nonmonotone perturbation theory. The quasilinear case, with $p \neq 2$, requires a strong restriction on the growth of the forcing term $|u|^{r-2} u$, which is caused by the loss of the elliptic estimate for the $p$-Laplacian operator defined by $\Delta_{p} u=\operatorname{div}\left(|\nabla u|^{p-2} \nabla u\right)($ see $[2])$.

Alaoui et al [12] considered the following nonlinear heat equation

$$
\left\{\begin{array}{l}
u_{t}-\operatorname{div}\left(|\nabla u|^{p(x)-2} \nabla u\right)=|u|^{r(x)-2} u+f, \quad \text { in } \Omega \times(0, T) \\
u(x, t)=0, \quad x \in \partial \Omega \times(0, T) \\
u(x, 0)=u_{0}(x) \text { in } \Omega
\end{array}\right.
$$

Where $\Omega$ is a bounded domain in $\mathbb{R}^{n}$ with smooth boundary $\partial \Omega$. Under suitable conditions on $r$ and $p$ and for $f=0$, they showed that any solution with nontrivial initial datum blows up in finite time. In the absence of the diffusion term in equation (1.1) when $p(x)=p$ and $r(x)=r$ proved the existence and plow up results have been established by many authors (See $[1-3,9,14,17])$. 
We should also point out that Polat [18] established a blow-up result for the solution with vanishing initial energy of the following initial boundary value problem

$$
u_{t}-u_{x x}+|u|^{m-2} u_{t}=|u|^{p-2} u .
$$

Where $m$ and $p$ are real constants.

In recent years, mush attention has been paid to the study of mathematical models of electro-theological fluids. This models inclode hyperbolic, parapolic or elliptic equations which are nonlinear with respect to the gradient of the thought solution with variable exponents of nonlinearity, (see $[4,5,10,15]$ ).

Our objective in this paper is to study: In the section 3, the blow up of the solutions of the problem (1.1) in the case $\omega=0$, in the section 4 , exponential growth of solution when $\omega>0$.

\section{Preliminaries}

We present in this section some Lemmas about the Lebesque and sobolev space with variables conponents (See $[6-8,12,13])$. Let $p: \Omega \rightarrow[1,+\infty]$ be a measurable function, where $\Omega$ is adomain of $\mathbb{R}^{n}$.

We define the Lebesque space with a variale exponent $p($.$) by$

$$
L^{p(.)}(\Omega):=\left\{v: \Omega \rightarrow \mathbb{R}: \text { measurable in } \Omega, A_{p(.)}(\lambda v)<+\infty, \text { for some } \lambda>0\right\},
$$

where $A_{p(.)}(v)=\int_{\Omega}|v(x)|^{p(x)} d x$.

The set $L^{p(.)}(\Omega)$ equipped with the norm ( Luxemburg's norm)

$$
\|v\|_{p(.)}:=\inf \left\{\lambda>0: \int_{\Omega}\left|\frac{v(x)}{\lambda}\right|^{p(x)} d x \leq 1\right\},
$$

$L^{p(\cdot)}(\Omega)$ is a Banach space [13].

We next, define the variable-exponent Sobolev space $W^{1, p(.)}(\Omega)$ as follows:

$$
W^{1, p(.)}(\Omega):=\left\{v \in L^{p(.)}(\Omega) \text { such that } \nabla v \text { exists and }|\nabla v| \in L^{p(.)}(\Omega)\right\}
$$

This is a Banach space with respect to the norm $\|v\|_{W^{1, p(.)}(\Omega)}=\|v\|_{p(.)}+\|\nabla v\|_{p(.)}$.

Furthmore, we set $W^{1, p(.)}(\Omega)$ to be the closure of $C_{0}^{\infty}(\Omega)$ in the space $W_{0}^{1, p(.)}(\Omega)$. Let us note that the space $W^{1, p(.)}(\Omega)$ has a differenet definition in the case of variable exponents.

However, under condition (1.3), both definitions are equivalent [13]. The space $W^{-1, p^{\prime}(.)}(\Omega)$, dual of $W_{0}^{1, p(.)}(\Omega)$, is defined in the same way as the classical Sobolev spaces, where $\frac{1}{p(.)}+\frac{1}{p^{\prime}(.)}=1$.

Lemma 2.1. (Poincaré's inequality) Let $\Omega \subset \mathbb{R}^{n}$ be a bounded domain and suppose that $p$ (.) satisfies (1.3), then

$$
\|v\|_{p(.)} \leq c\|\nabla v\|_{p(.)}, \quad \text { for all } v \in W_{0}^{1, p(.)}(\Omega)
$$


Where $c>0$ is a constant which depends on $p_{1}, p_{2}$, and $\Omega$ only. In particular, $\|\nabla v\|_{p(.)}$ define an equivalent norm on $W_{0}^{1, p(.)}(\Omega)$.

Lemma 2.2. If $p(.) \in C(\bar{\Omega})$ and $q: \Omega \rightarrow[1,+\infty)$ is a measurable function such that

$$
\text { essinf } \inf _{x \in \Omega}\left(p_{*}(x)-q(x)\right)>0 \text { with } p_{*}(x)=\left\{\begin{array}{l}
\frac{n p(x)}{\operatorname{ess\operatorname {sup}(n-p(x))}} \text { if } p_{2}<n \\
+\infty \quad \text { if } p_{2} \geq n
\end{array}\right.
$$

Then the embedding $W_{0}^{1, p(.)}(\Omega) \hookrightarrow L^{q(\cdot)}(\Omega)$ is continuous and compact.

Lemma 2.3. ( Hölder's Inequality) Suppose that $p, q, s \geq 1$ are measurable functions defined on $\Omega$ such that

$$
\frac{1}{s(y)}=\frac{1}{p(y)}+\frac{1}{q(y)}, \text { for a.e. } y \in \Omega .
$$

If $u \in L^{p(.)}(\Omega)$ and $v \in L^{q(\cdot)}(\Omega)$, then $u v \in L^{s(.)}(\Omega)$, with

$$
\|u v\|_{s(.)} \leq 2\|u\|_{p(.)}\|v\|_{q(.)}
$$

Lemma 2.4. If $p$ a measurable function on $\Omega$ satisfying (1.2), then we have

$$
\min \left\{\|u\|_{p(.)}^{p_{1}},\|u\|_{p(.)}^{p_{2}}\right\} \leq A_{p(.)}(u) \leq \max \left\{\|u\|_{p(.)}^{p_{1}},\|u\|_{p(.)}^{p_{2}}\right\}
$$

for any $u \in L^{p(.)}(\Omega)$.

\section{BLOW UP}

In this section, we prove that the solution of equation (1.1) blow up in finite time when $\omega=0$. we recall that (1.1), becomes

$$
\left\{\begin{array}{l}
u_{t}-\operatorname{div}\left(|\nabla u|^{p(x)-2} \nabla u\right)=b|u|^{r(x)-2} u \quad \text { in } \Omega \times(0, T) \\
u(x, t)=0, \quad x \in \partial \Omega, \quad t \geq 0 \\
u(x, 0)=u_{0}(x) \text { in } \Omega
\end{array}\right.
$$

We start with a local existence result for the problem (1.1), which is a direct result of the existence theorem by Agaki and Ôtani [2].

Proposition 3.1. For all $u_{0} \in W_{0}^{1, p(.)}(\Omega)$, there exists a number $T_{0} \in(0, T]$ such that the problem (1.1) has a solution $u$ on $\left[0, T_{0}\right]$ satisfying:

$$
u \in C_{w}\left(\left[0, T_{0}\right] ; W_{0}^{1, p(.)}(\Omega)\right) \cap C\left(\left[0, T_{0}\right], L^{r(.)}(\Omega)\right) \cap W^{1,2}\left(0, T_{0} ; L^{2}(\Omega)\right) .
$$

We define the energy functional associaeted of the problem (1.1)

$$
E(t)=\int_{\Omega} \frac{1}{p(x)}|\nabla u|^{p(x)} d x-b \int_{\Omega} \frac{1}{r(x)}|u|^{r(x)} d x .
$$


Theorem 3.1. Let the assumptions of proposition 1, be satisfied and assume that

$$
E(0)<0
$$

Then the solution of the problem (3.1), blow up in finite time.

Now, we let

$$
H(t):=-E(t)
$$

and

$$
L(t)=\frac{1}{2} \int_{\Omega} u^{2} d x
$$

To prove our result, we first establesh some Lemmas.

Lemma 3.1. Assume that (1.2) and (1.3), hold and $E(0)<0$. Then

$$
A_{p(.)}(\nabla u)<\frac{b p_{2}}{r_{1}} A_{r(.)}(u)
$$

and

$$
\frac{r_{1}}{b} H(0)<A_{r(.)}(u)
$$

Proof. We multiply the first equation of (3.1) by $u_{t}$ and integratying over the domain $\Omega$, we get

$$
\frac{d}{d t}\left(\int_{\Omega} \frac{1}{p(x)}|\nabla u|^{p(x)} d x-b \int_{\Omega} \frac{1}{r(x)}|u|^{r(x)} d x\right)=-\left\|u_{t}\right\|_{2}^{2},
$$

then

$$
E^{\prime}(t)=-\left\|u_{t}\right\|_{2}^{2} \leq 0
$$

Integrating $(3.8)$ over $(0, t)$, we obtain

$$
E(t) \leq E(0)<0
$$

By (3.2) and (3.9), we have

$$
\int_{\Omega} \frac{1}{p(x)}|\nabla u|^{p(x)} d x<b \int_{\Omega} \frac{1}{r(x)}|u|^{r(x)} d x,
$$

so that

$$
\int_{\Omega} \frac{1}{p_{2}}|\nabla u|^{p(x)} d x<\int_{\Omega} \frac{b}{r_{1}}|u|^{r(x)} d x .
$$

On the other hand, we have

$$
\begin{aligned}
H(t) & =-\int_{\Omega} \frac{1}{p(x)}|\nabla u|^{p(x)} d x+b \int_{\Omega} \frac{1}{r(x)}|u|^{r(x)} d x \\
& \leq b \int_{\Omega} \frac{1}{r(x)}|u|^{r(x)} d x .
\end{aligned}
$$


Then, by (3.10), (3.4) and (3.9), we obtain

$$
0<H(0)<H(t)<\frac{b}{r_{1}} A_{r(.)}(u) .
$$

Lemma 3.2. [16] Assume that (1.2), (1.3) hold and $E(0)<0$. Then the solution of (3.1), satisfies for some $c>0$

$$
A_{r(.)}(u) \geq c\|u\|_{r_{1}}^{r_{1}}
$$

Proof of theorem 1. We have

$$
\begin{aligned}
L^{\prime}(t) & =\int_{\Omega} u u_{t} d x \\
& =\int_{\Omega} u\left(\operatorname{div}\left(|\nabla u|^{p(x)-2} \nabla u\right)+b|u|^{r(x)-2} u\right) d x \\
& =-A_{p(.)}(\nabla u)+b A_{r(.)}(u) .
\end{aligned}
$$

Combining of $(3.12),(3.11)$ and $(3.6)$, leads to

$$
L^{\prime}(t) \geq c b\left(1-\frac{p_{2}}{r_{1}}\right)\|u\|_{r_{1}}^{r_{1}} .
$$

Now, we estimate $L^{\frac{r_{1}}{2}}(t)$, by the embedding of $L^{r_{1}}(\Omega) \hookrightarrow L^{2}(\Omega)$, we get

$$
L^{\frac{r_{1}}{2}}(t) \leq\left(\frac{1}{2}\|u\|_{r_{1}}^{2}\right)^{\frac{r_{1}}{2}} \leq c\|u\|_{r_{1}}^{r_{1}} .
$$

By combining (3.14) and (3.13), we obtain

$$
L^{\prime}(t) \geq \xi L^{\frac{r_{1}}{2}}(t)
$$

A direct integration of $(3.15)$, then yields

$$
L^{\frac{r_{1}}{2}-1}(t) \geq \frac{1}{L^{1-\frac{r_{1}}{2}}(0)-\xi t} .
$$

Therefore, $L$ blow up in a time $t^{*} \leq \frac{1}{L^{\frac{r_{1}}{2}-1}(0)}$.

\section{Exponential GROWTH}

In this section, we prove that the solution of equation (1.1) exponential growth when $\omega>0$.

Lemma 4.1. Suppose that (1.2) holds and $E(0)<0$. Then,

$$
\int_{\Omega}|u|^{m(x)} d x \leq c\left(\|u\|_{r_{1}}^{r_{1}}+H(t)\right) .
$$


Proof.

$$
\int_{\Omega}|u|^{m(x)} d x=\int_{\Omega_{-}}|u|^{m(x)} d x+\int_{\Omega_{+}}|u|^{m(x)} d x,
$$

where

$$
\Omega_{+}=\{x \in \Omega /|u(x, t)| \geq 1\} \text { and } \Omega_{-}=\{x \in \Omega /|u(x, t)|<1\} .
$$

So, we get

$$
\begin{aligned}
\int_{\Omega}|u|^{m(x)} d x & \leq c\left[\left(\int_{\Omega_{-}}|u|^{r_{1}} d x\right)^{\frac{m_{1}}{r_{1}}}+\left(\int_{\Omega+}|u|^{r_{1}} d x\right)^{\frac{m_{2}}{r_{1}}}\right] \\
& \leq c\left(\|u\|_{r_{1}}^{m_{1}}+\|u\|_{r_{1}}^{m_{2}}\right) .
\end{aligned}
$$

Exploiting the algebric inequality

$$
z^{v} \leq(z+1) \leq\left(1+\frac{1}{a}\right)(z+a), \forall z>0,0<v \leq 1, a \geq 0
$$

we have

$$
\begin{aligned}
\|u\|_{r_{1}}^{m_{1}} & \leq c\left(\|u\|_{r_{1}}^{r_{1}}\right)^{\frac{m_{1}}{r_{1}}} \leq c\left(1+\frac{1}{H(0)}\right)\left(\|u\|_{r_{1}}^{r_{1}}+H(0)\right) \\
& \leq c\left(\|u\|_{r_{1}}^{r_{1}}+H(t)\right) .
\end{aligned}
$$

Similarly,

$$
\begin{aligned}
\|u\|_{r_{1}}^{m_{2}} & \leq c\left(\|u\|_{r_{1}}^{r_{1}}\right)^{\frac{m_{2}}{r_{1}}} \leq c\left(1+\frac{1}{H(0)}\right)\left(\|u\|_{r_{1}}^{r_{1}}+H(0)\right) \\
& \leq c\left(\|u\|_{r_{1}}^{r_{1}}+H(t)\right) .
\end{aligned}
$$

This gives

$$
\int_{\Omega}|u|^{m(x)} d x \leq c\left(\|u\|_{r_{1}}^{r_{1}}+H(t)\right) .
$$

Theorem 4.1. Let the assumptions of proposition 1, be satisfied and assume that (3.3) holds. Then the solution of the problem (1.1), grows exponentially.

Proof. By the same procedure of the proof the Lemma 5, we get

$$
E^{\prime}(t)=-\left\|u_{t}\right\|_{2}^{2}-\omega \int_{\Omega}|u|^{m(x)-2} u_{t}^{2} \leq 0,
$$

then, we have

$$
H^{\prime}(t)=\left\|u_{t}\right\|_{2}^{2}+\omega \int_{\Omega}|u|^{m(x)-2} u_{t}^{2} d x \geq 0 .
$$


We define

$$
G(t)=H(t)+\epsilon L(t)
$$

for $\epsilon$ small to be chosen later.

The time derivative of (4.4), we obtain

$$
G^{\prime}(t)=H^{\prime}(t)+\epsilon \int_{\Omega} u u_{t} d x
$$

By using (1.1), we get

$$
G^{\prime}(t)=H^{\prime}(t)-\epsilon A_{p(.)}(\nabla u)+\epsilon b A_{r(.)}(u)-\epsilon \omega \int_{\Omega}|u|^{m(x)-2} u_{t} u d x .
$$

To estimate the last term in the right hand side of (4.5), by using the following Young's Inequality

$$
\begin{aligned}
& X Y \leq \delta X^{2}+\delta^{-1} Y^{2}, \quad X, Y \geq 0, \delta>0 . \\
& \int_{\Omega}|u|^{m(x)-2} u_{t} u d x=\int_{\Omega}|u|^{\frac{m(x)-2}{2}} u_{t}|u|^{\frac{m(x)-2}{2}} u d x \\
& \leq \delta \int_{\Omega}|u|^{m(x)-2} u_{t}^{2} d x+\delta^{-1} \int_{\Omega}|u|^{m(x)} d x .
\end{aligned}
$$

We conclude

$$
\begin{aligned}
G^{\prime}(t) \geq & (1-\epsilon \delta) \int_{\Omega}|u|^{m(x)-2} u_{t}^{2} d x+\left\|u_{t}\right\|_{2}^{2}-\epsilon A_{p(.)}(\nabla u) \\
& +\epsilon b A_{r(.)}(u)-\epsilon \omega \delta^{-1} \int_{\Omega}|u|^{m(x)} d x .
\end{aligned}
$$

Then

$$
\begin{aligned}
G^{\prime}(t) \geq & (1-\epsilon \delta) \int_{\Omega}|u|^{m(x)-2} u_{t}^{2} d x+\left\|u_{t}\right\|_{2}^{2}-\epsilon \omega \delta^{-1} \int_{\Omega}|u|^{m(x)} d x \\
& +\epsilon(1-\mu) r_{1} H(t)+\epsilon b \mu A_{r(.)}(u)+\epsilon\left((1-\mu) \frac{r_{1}}{p_{2}}-1\right) A_{p(.)}(\nabla u),
\end{aligned}
$$

where $\mu$ is a constant such that $0<\mu \leq 1-\frac{p_{2}}{r_{1}}$.

Also, by using (3.6), we obtain

$$
\begin{aligned}
G^{\prime}(t) \geq & (1-\epsilon \delta) \int_{\Omega}|u|^{m(x)-2} u_{t}^{2} d x+\left\|u_{t}\right\|_{2}^{2}-\epsilon \omega \delta^{-1} \int_{\Omega}|u|^{m(x)} d x \\
& +\epsilon(1-\mu) r_{1} H(t)+\epsilon\left(b \mu+1-\mu-\frac{p_{2}}{r_{1}}\right) A_{r(.)}(u) .
\end{aligned}
$$


Then, by Lemma 7 and (3.11), (4.7) becomes

$$
\begin{aligned}
G^{\prime}(t) \geq & (1-\epsilon \delta) \int_{\Omega}|u|^{m(x)-2} u_{t}^{2} d x+\left\|u_{t}\right\|_{2}^{2}-\epsilon c \omega \delta^{-1}\left(\|u\|_{r_{1}}^{r_{1}}+H(t)\right) \\
& +\epsilon(1-\mu) r_{1} H(t)+\epsilon c\left(b \mu+1-\mu-\frac{p_{2}}{r_{1}}\right)\|u\|_{r_{1}}^{r_{1}}
\end{aligned}
$$

So that

$$
\begin{aligned}
G^{\prime}(t) \geq & (1-\epsilon \delta) \int_{\Omega}|u|^{m(x)-2} u_{t}^{2} d x+\left\|u_{t}\right\|_{2}^{2}+\epsilon\left((1-\mu) r_{1}-c \omega \delta^{-1}\right) H(t) \\
& +\epsilon\left(c\left(b \mu+1-\mu-\frac{p_{2}}{r_{1}}\right)-c \omega \delta^{-1}\right)\|u\|_{p_{1}}^{p_{1}}
\end{aligned}
$$

So, we chosen $\delta$ large sufficient and $\epsilon$ small enough for that we can find $\lambda_{1}, \lambda_{2}>0$, such that

$$
G^{\prime}(t) \geq \lambda_{1} H(t)+\lambda_{2}\|u\|_{r_{1}}^{r_{1}} \geq K_{1}\left(H(t)+\|u\|_{r_{1}}^{r_{1}}\right)
$$

and

$$
G(0)=H(0)+\epsilon L(0)>0
$$

Similarly in (4.7), we have

$$
\|u\|_{2}^{2} \leq c\left(H(t)+\|u\|_{r_{1}}^{r_{1}}\right) .
$$

On the other hand, by (4.11), we get

$$
G(t) \leq K_{2}\left(H(t)+\|u\|_{r_{1}}^{r_{1}}\right)
$$

Combining with (4.12) and (4.10), we arrive at

$$
G^{\prime}(t) \geq \eta G(t)
$$

Finally, a simple integration of (4.13) gives

$$
G(t) \geq G(0) e^{\eta t}, \quad \forall t \geq 0
$$

Thus completes the proof.

\section{REFERENCES}

[1] G. Akagi, Local existence of solutions to some degenerate parabolic equation associated with the p-Laplacian, J. Differential Equations 241 (2007), 359-385.

[2] G. Akagi and M. Ôtani, Evolutions inclusions governed by subdifferentials in reflexive Banach spaces, J. Evol. Equ. 4 (2004), 519-541.

[3] G. Akagi and M. Ôtani, Evolutions inclusions governed by the difference of two subdifferentials in reflexive Banach spaces, J. Differential Equations 209 (2005), 392-415.

[4] S.N. Antontsev and V. Zhikov, Higher integrability for parabolic equations of $p(x, t)$-Laplacian type. Adv. Differ. Equ. 10 (2005), 1053-1080. 
[5] Y. Chen, S. Levine and M. Rao, Variable exponent, linear growth functions in image restoration. SIAM J. Appl. Math. 66 (2006), 1383-1406.

[6] D. Edmunds and J. Rakosnik, Sobolev embeddings with variable exponent, Stud. Math. 143 (3) (2000), $267-293$.

[7] D. Edmunds and J. Rakosnik Sobolev embeddings with variable exponent. II, Math. Nachr. 246 (1) (2002), 53-67.

[8] X. Fan and D. Zhao, On the spaces $L^{p(x)}(\Omega)$ and $W^{m, p(x)}(\Omega)$, J. Math. Anal. Appl. 263 (2) (2001), $424-446$.

[9] H. Fujita, On the blowing up solutions of the Cauchy problem for $u_{t}=\Delta u+u^{1+\alpha}$, J. Fac. Sci. Univ. Tokyo Sect. A.Math. 16 (1966), 105-113.

[10] Y. Gao, B. Guo and W.Gao, Weak solutions for a high-order pseudo-parabolic equation with variable exponents. Appl. Anal. 93 (2) (2014), 322-338.

[11] Z. Jiang, S. Zheng, and X. Song, Blow-up analysis for a nonlinear diffusion equation with nonlinear boundary conditions, Appl. Math. Lett. 17 (2) (2004), 193-199.

[12] A.M. Kbiri, S.A. Messaoudi. and H.B. Khenous, A blow-up result for nonlinear generalized heat equation, Comput. Math. Appl. 68 (12) (2014), 1723-1732.

[13] D. Lars, P. Harjulehto, P. Hasto and M. Ruzicka, Lebesgue and Sobolev spaces with variable exponents, in: Lecture Notes in Mathematics, Springer, 2011.

[14] J. Leray and J.L.Lions, Quelques résultats de Visick sur les problémes elliptiques non linéaires pour les méthodes de Minty-Browder, Bull. Soc. Math. France 93 (1965), 97-107.

[15] S.Z. Lian, W.J. Gao, CL. Cao and HJ. Yuan, Study of the solutions to a model porous medium equation with variable exponents of nonlinearity. J. Math. Anal. Appl. 342 (2008), 27-38.

[16] S.A. Messaoudi and A. Talahmeh, Blowup in solutions of a quasilinear wave equation with variable-exponent nonlinearities, Math. Methods Appl. Sci. 40 (18) (2017), 6976-6986.

[17] M. Ôtani, Nonmonotone perturbations for nonlinear parabolic equations associated with subdifferential operators, Cauchy problems, J. Differential Equations 46 (1982), 268-299.

[18] N. Polat, Blow up of solution for a nonlinear reaction diffusion equation with multiple nonlinearities, Int. J. Sci. Technol. 2 (2) (2007), 123-128. 